\title{
Complex Optimization Problems in Locational Analysis and Scheduling
}

\author{
Xavier Delorme • Alexandre Dolgui • \\ Alexander Kolokolov
}

Published online: 1 August 2012

(C) Springer Science+Business Media B.V. 2012

This issue is composed of updated and extended versions of seven papers selected at the IFAC Symposiums INCOM06 and INCOM09. All the papers passed through a rigorous peer review process with at least 3 referees each. This was performed twice: once before the symposium, for their initial version, and again after the symposium, for the extended version.

The 12th and 13th IFAC symposiums INCOM06 (www.emse.fr/incom06) and INCOM09 (incom09.org) were major scientific events on Operations Research and their applications for production management with large academic and industrial audiences. The articles for this issue are from the long-established symposium sessions "Locational analysis" and "Theoretical aspects of scheduling" where the talks were focused on complex combinatorial optimisation problems and innovative algorithmic approaches for their solution. When selecting these papers, we were especially receptive to new applications of combinatorial optimization and novel or not well enough known approaches employed to solve hard problems in this domain. We hope that the readers will find here excellent examples of effective methods as well as key sources for inspiration.

\footnotetext{
X. Delorme · A. Dolgui $(\varangle)$

Henri Fayol Institute, Ecole des Mines de Saint-Étienne, UMR CNRS 6158, LIMOS, 158, cours Fauriel, 42023 Saint-Étienne, France

e-mail: dolgui@emse.fr

URL: www.emse.fr/ dolgui

X. Delorme

e-mail: delorme@emse.fr

URL: www.emse.fr/ delorme
}

A. Kolokolov

Omsk Branch of Sobolev Institute of Mathematics of Siberian

Branch of Russian Academy of Sciences, 13, Pevtsov St., 644043 Omsk, Russia

e-mail: kolo@ofim.oscsbras.ru 
The first paper deals with "Solving a bi-criteria problem of optimal service centers location" by Alexander A. Kolokolov and Lidia A. Zaozerskaya. The authors consider the problem of service centres location as a bi-criteria dominating set problem in which a total cost of the centres location is minimized and a service level (satisfaction of requirements) is maximized. They used an integer linear program for this problem combined with the L-partition approach to solve it. The tradeoff method, decomposition and L-class enumeration algorithm were used to find a subset of the Pareto-optimal set. The results of a computational experiment are presented. The L-partition approach is not well known but seems to be appropriate for a large spectrum of combinatorial optimization problems.

The second paper "Equipment location in machining transfer lines with multispindle heads" by Olga Battaïa et al., examines an industrial problem of equipment selection and location. To solve this problem in an acceptable computational time, the authors propose several bounds and sophisticated pre-processing procedures decreasing the size of the problem and so shorten the solution time. This approach of a model reduction based on analysing and transforming its constraints seems to be very promising and should be suggested for many other line design and resource location problems.

Next paper "Clustering of patients' trajectories with an auto-stopped bisecting K-medoids algorithm" by Hongying Fei and Nadine Meskens addresses a problem related to classification of patient trajectories within a hospital. The authors express this as a sequence clustering problem and present an algorithm based on Bisecting K-Medoids to deal with it. Results obtained with real data are inspiring.

In the fourth paper "Complexity of buffer capacity allocation problems for production lines with unreliable machines" by Alexandre Dolgui, Anton Eremeev, Mikhail Y. Kovalyov and Viatcheslav Sigaev the authors consider a well know problem of buffer allocation in production lines but attack a yet unstudied aspect: analysis of its combinatorial complexity. Often in literature only line performance was evaluated. Here, the optimisation problem is considered which consists of determining the sizes of buffers between machines optimizing a given criterion. The authors show that even for basic cases this problem is NP-hard. This fundamental result gives a new light on this large domain of scientific research.

In "A goal programming model for staff scheduling at a telecommunication center" Mohamed Aly O. Louly studies an employee scheduling problem that occurs in telecommunications centres. In addition to the numerous constraints due to requirements to fulfil, the main difficulty of this problem comes from the need to take fairness into account. The approach proposed is based on a goal programming model. Results obtained on a real-life application are presented and discussed. This is a new and interesting application of rigorous techniques used for human resource management.

The paper "Scheduling personal finances via integer programming" by Oliver Braun and Yuri N. Sotskov presents an original scheduling problem related to the planning of personal finances. Their approach is based on an integer program which seeks to determine the optimal dates to buy or sell assets according to an individual's preferences. The results presented illustrate that this model can solve instances corresponding to real-size problems and can perform a stability analysis. This model opens novel applications for combinatorial optimisation. In addition, the approach used for stability analysis is very attractive. 
Finally, the last paper is "Analysis and solving SAT and MAX-SAT problems using an L-partition approach" by Alexander Kolokolov, Alexander Adelshin and Darya Yagofarova where the authors study these standard problems using the integer linear programs and L-partition approach. This theoretical approach can be useful for many discrete optimization problems including location, covering and scheduling. Some examples of SAT and MAX-SAT families for which the cardinality of L-covering for the relaxation polytope grows exponentially with the number of variables are pointed out. This property is helpful in analysis and development of algorithms based on a linear relaxation.

We hope that all our readers will enjoy and take away some insights and knowledge from the papers presented.

We would like to thank the authors for their contributions and are very grateful to all the anonymous referees who gave their time. Thanks as well to Prof. Victor J. Rayward-Smith, Editor-in-Chief of the Journal of Mathematical Modelling and Algorithms for Operations Research for its help in publishing this special issue. The Guest Editors thank also Chris Yukna for his help in English. 